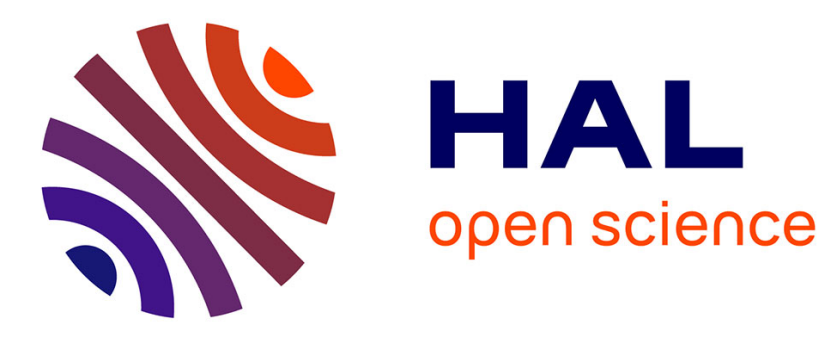

\title{
Spectral dependency of superconducting single photon detectors
}

\author{
Laurent Maingault, M. Tarkhov, I. Florya, A. Semenov, Roch Espiau de \\ Lamaestre, Paul Cavalier, G. Gol'Tsman, Jean-Philippe Poizat, Jean-Claude \\ Villegier
}

\section{To cite this version:}

Laurent Maingault, M. Tarkhov, I. Florya, A. Semenov, Roch Espiau de Lamaestre, et al.. Spectral dependency of superconducting single photon detectors. Journal of Applied Physics, 2010, 107, pp.116103. 10.1063/1.3374636 . hal-00998846

\section{HAL Id: hal-00998846 https://hal.science/hal-00998846}

Submitted on 3 Jun 2014

HAL is a multi-disciplinary open access archive for the deposit and dissemination of scientific research documents, whether they are published or not. The documents may come from teaching and research institutions in France or abroad, or from public or private research centers.
L'archive ouverte pluridisciplinaire HAL, est destinée au dépôt et à la diffusion de documents scientifiques de niveau recherche, publiés ou non, émanant des établissements d'enseignement et de recherche français ou étrangers, des laboratoires publics ou privés. 


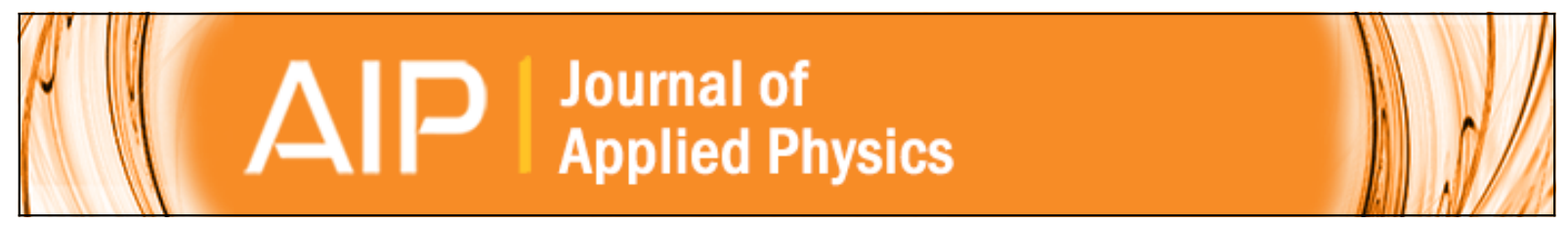

\section{Spectral dependency of superconducting single photon detectors}

L. Maingault, M. Tarkhov, I. Florya, A. Semenov, R. Espiau de Lamaëstre, P. Cavalier, G. Gol'tsman, J.-P. Poizat , and J.-C. Villégier

Citation: Journal of Applied Physics 107, 116103 (2010); doi: 10.1063/1.3374636

View online: $\mathrm{http}: / / \mathrm{dx}$.doi.org/10.1063/1.3374636

View Table of Contents: http://scitation.aip.org/content/aip/journal/jap/107/11 ?ver=pdfcov

Published by the AIP Publishing

\section{Articles you may be interested in}

Spatial dependence of output pulse delay in a niobium nitride nanowire superconducting single-photon detector Appl. Phys. Lett. 98, 201116 (2011); 10.1063/1.3581054

Low noise superconducting single photon detectors on silicon

Appl. Phys. Lett. 93, 131101 (2008); 10.1063/1.2990646

A cascade switching superconducting single photon detector

Appl. Phys. Lett. 91, 262509 (2007); 10.1063/1.2828138

Kinetic-inductance-limited reset time of superconducting nanowire photon counters

Appl. Phys. Lett. 88, 111116 (2006); 10.1063/1.2183810

Picosecond superconducting single-photon optical detector

Appl. Phys. Lett. 79, 705 (2001); 10.1063/1.1388868

\section{AlP Re-register for Table of Content Alerts}

\section{Create a profile.

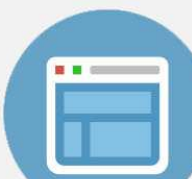 \\ Sign up today!}




\title{
Spectral dependency of superconducting single photon detectors
}

\author{
L. Maingault, ${ }^{1, a)}$ M. Tarkhov ${ }^{2,3}$ I. Florya, ${ }^{2}$ A. Semenov, ${ }^{2}$ R. Espiau de Lamaëstre, ${ }^{4}$ \\ P. Cavalier, ${ }^{1}$ G. Gol'tsman, ${ }^{3}$ J.-P. Poizat, ${ }^{3}$ and J.-C. Villégier ${ }^{1}$ \\ ${ }^{1}$ SPSMS, UMR-E 9001, CEA-INAC/UJF, 38054 Grenoble, France \\ ${ }^{2}$ Moscow State Pedagogical University, 119991 Moscow, Russia \\ ${ }^{3}$ CEA-CNRS-UJF Group Nanophysique et Semiconducteurs, Institut Néel, 38042 Grenoble, France \\ ${ }^{4}$ CEA LETI MINATEC, 38054 Grenoble, France
}

(Received 10 September 2009; accepted 2 March 2010; published online 4 June 2010)

\begin{abstract}
We investigate the effect of varying both incoming optical wavelength and width of $\mathrm{NbN}$ nanowires on the superconducting single photon detectors (SSPD) detection efficiency. The SSPD are current biased close to critical value and temperature fixed at $4.2 \mathrm{~K}$, far from transition. The experimental results are found to verify with a good accuracy predictions based on the "hot spot model," whose size scales with the absorbed photon energy. With larger optical power inducing multiphoton detection regime, the same scaling law remains valid, up to the three-photon regime. We demonstrate the validity of applying a limited number of measurements and using such a simple model to reasonably predict any SSPD behavior among a collection of nanowire device widths at different photon wavelengths. These results set the basis for designing efficient single photon detectors operating in the infrared (2-5 $\mu \mathrm{m}$ range). (C) 2010 American Institute of Physics. [doi:10.1063/1.3374636]
\end{abstract}

Superconducting single photon detectors (SSPD) (Ref. 1) offer great opportunities for single photon detection in the near infrared (IR) telecom wavelengths (1.3 and $1.55 \mu \mathrm{m})$ where semiconducting avalanche photodiodes ${ }^{2}$ usually suffer from rather high dark counts. High detection efficiency (DE), with photonics designs in the detector environment, ${ }^{3-5}$ ultrafast reset time, ${ }^{6}$ as well as photon number resolution ${ }^{7}$ have already been achieved. This type of detector has already brought major achievements in quantum communication ${ }^{8,9}$ and should lead to devices for compact optical spectroscopy. ${ }^{10}$ Thanks to small superconducting gaps SSPD can in principle operate very far in the IR. ${ }^{11}$ Implementation of SSPD operating above $2 \mu \mathrm{m}$ would open the possibility of single photon counting in this spectral range, (currently not covered by existing photon counters), which would be a significant step forward for applications such as light detection and ranging, atmospheric gas spectroscopy, medical imaging or astronomy.

One intuitive detection mechanism which is still under discussion is the hot-spot model ${ }^{12}$ : a "hot-spot" is created by the absorption of a single photon in a superconducting nanowire etched out of a thin film (made of $\mathrm{NbN}$ in our case). Owing to the very small thickness $(t<5 \mathrm{~nm})$ of the film, the area of the hot-spot is proportional to the energy of the photon. If this hot-spot is big enough, it triggers the transition of the whole nanowire cross-section into a resistive state, leading to a measurable voltage pulse. The authors of Ref. 12 theoretically explained the hot-spot detection mechanism with deep-insight into superconductivity and heat diffusion behavior. They experimentally checked this model for different wavelengths. ${ }^{13,14}$

In this communication, we further test the validity of the hot-spot model $^{15}$ by an extensive experimental investigation

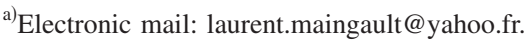

of SSPD detection efficiencies for different nanowire widths and incoming photon wavelengths. The extrapolation to the IR of the scaling law we have found paves the road to the design of single photon detectors in this spectral domain.

In the hot-spot model, a photon is detected only if the hot-spot is large enough, as compared to the width $w$ of the nanowire, for the current density on the side of this hot-spot to locally overcome the critical current density, $J_{c}$ [see Fig. 1(a)]. In a geometrical point-of-view, this condition writes

$$
D>w\left(1-I / I_{c}\right),
$$

where $D$ is the hot-spot diameter, $w$ is the nanowire width and $I$ the current flowing in the nanowire. Furthermore, the hot-spot surface area, $\pi D^{2} / 4$, scales as the photon energy, $h c / \lambda$, where $\lambda$ is the wavelength of the incoming light. The diameter of the hot-spot is proportional to $1 / \sqrt{\lambda}$. Therefore, for a given film thickness $t$ and a given normalized bias current $I / I_{c}$, DE should exhibit a cut-off at a given value of the reduced parameter $w \sqrt{\lambda}$. This condition reads:

$$
w \sqrt{\lambda}<K_{1}
$$

where $K_{1}$ is a constant.

In order to test the latter acting law of the cut-off [Eq. (2)], we fabricated $\mathrm{NbN}$ SSPDs with different nanowire widths (from 80 up to $480 \mathrm{~nm}$ ), the nanowire being folded on a surface of $10 \times 10 \mu \mathrm{m}^{2}$ with a filling factor ranging from 0.4 to 0.6 and a length from 200 to $600 \mu \mathrm{m}$. These SSPDs are made of $\mathrm{NbN}$ films of thickness $4.4 \mathrm{~nm}$ sputtered on a R-plane sapphire substrate. ${ }^{16} \mathrm{NbN}$ nanowires are patterned with an electron-beam lithography, which is the most critical step of the process. This fabrication process is completely described in Refs. 4 and 17. Various laser diodes were used for the different excitation wavelengths. All DE were measured with a bias current of $I=0.96 I_{c}$ at $\mathrm{T}=4.2 \mathrm{~K}$, the critical density current, $j_{c}=I_{c} /(w t)$ being in a $3-7 \times 10^{-10} \mathrm{~A} / \mathrm{m}^{2}$ 

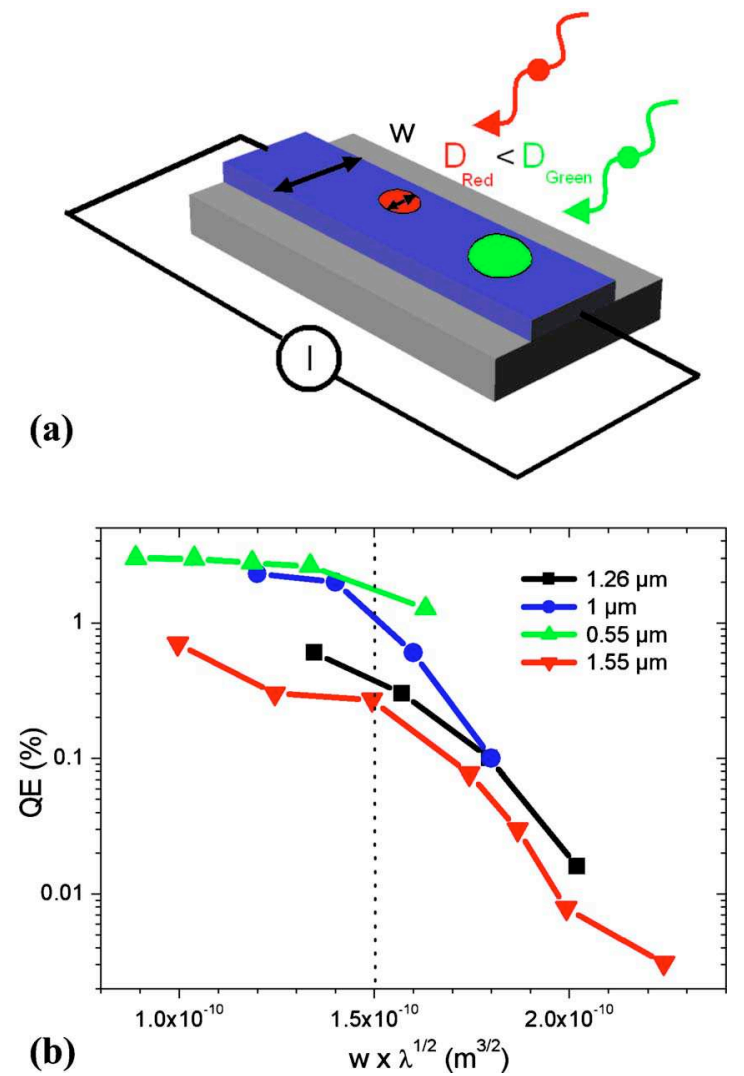

FIG. 1. (Color online) (a) Scheme of the hot-spot. The NbN stripe is in blue (b) Dependence of normalized DE vs reduced parameter $w \sqrt{\lambda}$ at different wavelengths. This was measured with $10 \times 10 \mu \mathrm{m}^{2}$ SSPDs with width varying from 120 to $220 \mathrm{~nm}$.

range. This corresponds to an optimal working point with a dark count rate for all samples around 10 counts/s. DE is defined as the ratio of the detection pulse rate over the incoming photon flux. The latter is measured with a calibrated semiconductor power meter.

Inset of Fig. 2 shows a measurement of the DE as a function of the nanowire width for a fixed wavelength $\lambda=1.55 \mu \mathrm{m}$. As expected, there is a specific nanowire width, that we estimate of about $130 \mathrm{~nm}$, above which DE

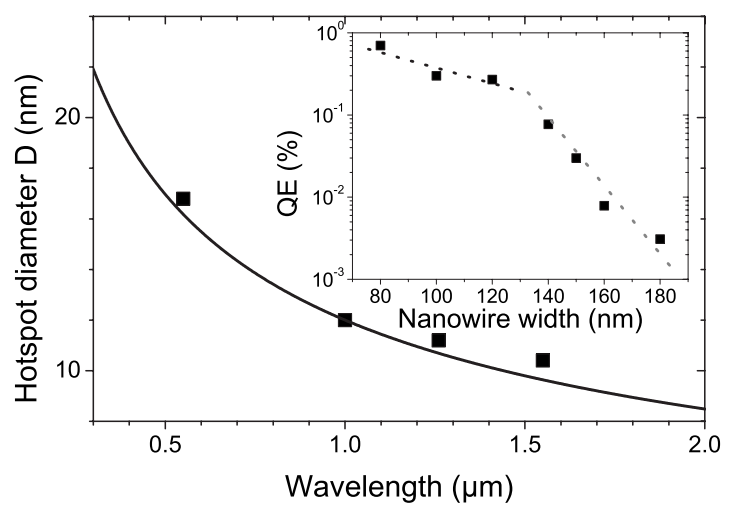

FIG. 2. Hot spot diameter $D$ for a single photon of wavelength $\lambda$. This diameter is obtained from the largest nanowire width that gives a measurable voltage pulse under a given bias current [cf. Eq. (1)]. The line is $K_{1} / \sqrt{\lambda}$ with $K_{1}$ being the constant extracted from the data shown in Fig. 1. Inset is an example of the determination of the minimum nanowire width $w_{c}(\approx 130 \mathrm{~nm}$ here $)$ for detection at a wavelength $\lambda=1.55 \mu \mathrm{m}$. Dotted lines are guides to the eye.

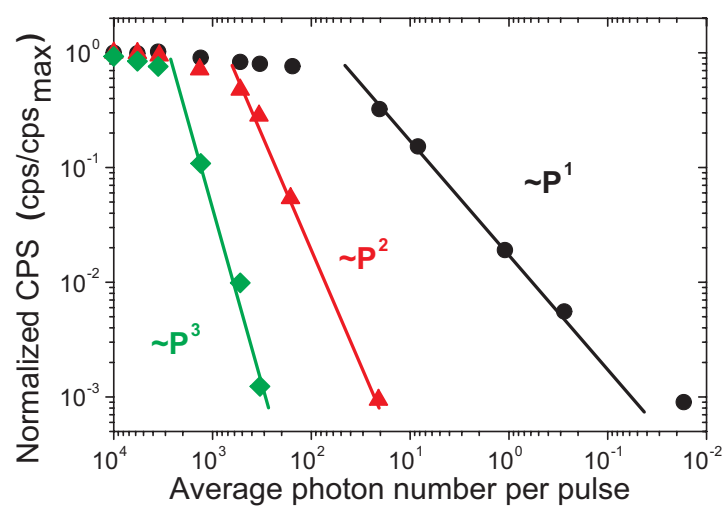

FIG. 3. (Color online) Dependence of normalized counts per second vs incoming light power. Circles (black), triangles (red) and diamond (green) symbols, respectively, correspond respectively to a linear, quadratic $\left(\propto P^{2}\right)$ and cubic $\left(\propto P^{3}\right)$ dependence and thus to single, two, and three-photon regime because the counts per seconds are proportional to $P^{n}$, where $P$ is the light power and $n$ the number of photons detected.

drops rapidly. Above this threshold, the detection mechanism changes and is most likely due to thermodynamic unbinding of vortex-antivortex pairs $^{18}$ or phase-slip centers generation. ${ }^{19,20}$ This mechanism is much less efficient so that DE decreases rapidly for wider nanowires. This observed behavior is very similar to what is observed when either wavelength ${ }^{13}$ or bias current ${ }^{11}$ is varied, in agreement with the prediction of the hot-spot model. In this work, we go beyond the single parameter threshold behavior by verifying the scaling law given in Eq. (2). Extensive DE measurements as a function of both wavelength and nanowire width were undertaken. Figure 1 displays the variation of DE for four different wavelengths as a function of the normalized parameter $w \sqrt{\lambda}$. A unique threshold value $K_{1}=1.5 \times 10^{-10} \mathrm{~m}^{3 / 2}$ for the whole set of data, in agreement with Eq. (2), is observed.

Moreover, the diameter of the hot-spot can be easily calculated within this hot-spot framework. The critical nanowire width $w_{c}$ at a given wavelength $\lambda$ gives the hot-spot diameter by a combination of Eqs. (1) and (2): $D(\lambda)=w_{c}\left(1-I / I_{c}\right)$. This is shown in Fig. 2.

The validity of the hot-spot model can be further tested in the multiphoton detection regime. Indeed, above this threshold, the energy of a single photon is not enough to trigger a resistive transition of the detector. In order to become resistive, the nanowire should be hit by more than one photon: this is the multiphoton regime. More precisely, it is possible to know in which regime the detector is by measuring the slope of the DE versus light power plot. ${ }^{1,21}$ For a mean number of $m$ photons per pulse, the probability $P(n)$ of absorbing $n$ photons from a given pulse is given by the Poisson distribution. For very weak $(m \ll 1)$ photon fluxes, the probability of detecting one photon, two photons, and three photons is

$$
P(1) \approx m, \quad P(2) \approx \frac{m^{2}}{2}, \quad P(3) \approx \frac{m^{3}}{6}, \text { etc. }
$$

Figure 3 shows the dependence of counts per second versus light power for single, two, and three-photon regime. Since the expected curve scales as $P^{n}$, where $P$ is the incoming light power and $n$ the number of photons needed for detection, the curve slope is a direct indication of the number of photons needed to trigger a detection. We have performed 


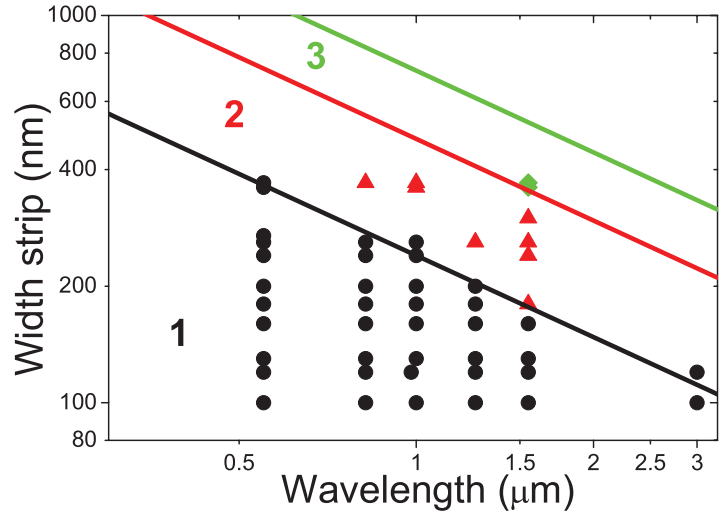

FIG. 4. (Color online) Photon regime dependence with wavelength and nanowire width. Black circles correspond to single-photon regime, red triangles to two-photon regime and green diamonds to three-photon regime. Lines are the expected borderlines from a regime to another, with a $(-1 / 2)$ slope.

such power dependency plots for all possible $(w, \lambda)$ values. The result is shown in Fig. 4. Different regions can be clearly identified and correspond to $n=1,2$, or 3 photon regimes. The border between the $n=i, n=i+1$ regions are $w \sqrt{\lambda}=K_{i}^{\prime}$ curves, where $K_{i}^{\prime}$ is a constant. As the nanowire width or the photon wavelength increases the relative size of the hot-spot with respect to the nanowire width becomes too small to allow for single photon detection: one enters the two, and then three-photon regimes (cf. Fig. 4). In the two-photon regime, Eq. (1) becomes $2 D>w\left(1-I / I_{c}\right)$. This corresponds to $K_{2}^{\prime}=2 K_{1}^{\prime}$, which indicates that for a given wavelength the two-photon regime cut-off occurs for a nanowire width twice as large as for the single photon cut-off. We also point out that a decent fit is obtained for a value $K_{1}^{\prime}=2.0$ $\times 10^{-10} \mathrm{~m}^{3 / 2}$, close to the value $K_{1}$ obtained from Fig. 1 .

$\mathrm{DE}(w, \lambda)$ scaling experiments in the single photon and multiphoton regimes have both shown a similar threshold: $K_{1} \approx K_{1}^{\prime}$. This experimental value is compared below to the expected value obtained in the framework of the hot-spot model. We assume that when the normal area is formed, nonequilibrium quasiparticles created after the photon absorption are trapped in due to the normal-superconducting interface. The number of quasiparticles can be estimated as $N_{Q P}^{t h}=h c /(\lambda \epsilon)$, where the characteristic energy of quasiparticles, $\epsilon$, is about $k_{B} T_{c}$ (with $k_{B}$, the Boltzmann constant and $T_{c}$, the critical temperature of the nanowire). On the other hand, when the superconducting gap inside the spot is suppressed, the quasiparticle concentration is $N_{0} k_{B} T_{c}$, where $N_{0}$ is the density of states at Fermi level. So the theoretical hot-spot volume is $V=N_{Q P}^{\text {th }} /\left(N_{0} k_{B} T\right)=h c /\left(\lambda N_{0} k_{B}^{2} T_{c}^{2}\right)$. Comparing this value with the threshold diameter $D=w\left(1-I / I_{c}\right)$, we come to the following threshold value:

$$
w \sqrt{\lambda}=\frac{2}{\left(1-I / I_{c}\right)} \sqrt{\frac{h c}{\pi N_{0} k_{B}^{2} T_{c}^{2} t}}
$$

with $t$ the film thickness. Substituting our NbN films parameters $\left(T_{c}=12.3 \mathrm{~K}, N_{0}=1.2 \times 10^{47} \mathrm{~m}^{-3} \mathrm{~J}^{-1}, t=4.4 \mathrm{~nm}\right)$, we obtain $K_{1}^{\text {th }}=3.1 \times 10^{-9} \mathrm{~m}^{3 / 2}$. This value is higher than what we obtained experimentally. This may be attributed to energy and quasiparticle losses during the hot-spot formation. Quantitatively, an internal efficiency of quasiparticles creation, $\eta$, can be determined as $\eta=N_{Q P}^{\text {meas }} / N_{Q P}^{\text {th }}=0.5 \%$. The quality of our films and/or lithography could be one of the reasons of this poor efficiency.

In conclusion we have checked experimentally that the hot-spot picture gives a good qualitative description of the SSPD detection mechanism. When measuring SSPD efficiencies with different nanowire widths, we found a threshold well explained by the hot-spot model. The DE drops quickly when $w \sqrt{\lambda}>2.0 \times 10^{-10} \mathrm{~m}^{3 / 2}$. Efficient IR detectors will therefore require narrow nanowires and their fabrication will be technologically very demanding. Our result sets the minimum nanowire width limit for designing single photon detectors in the far IR range.

This work was supported by European projects FP6 STREP "SINPHONIA" (Contract No. NMP4-CT-200516433) and IP “QAP” (Contract No. 15848).

${ }^{1}$ G. N. Gol'tsman, O. Okunev, G. Chulkova, A. Lipatov, A. Semenov, K. Smirnov, B. Voronov, A. Dzardanov, C. Williams, and R. Sobolewski, Appl. Phys. Lett. 79, 705 (2001).

${ }^{2}$ D. Renker and E. Lorenz, J. Instrumentation 4, P04004 (2009).

${ }^{3}$ K. M. Rosfjord, J. K. W. Yang, E. A. Dauler, A. J. Kerman, V. Anant, B. M. Voronov, G. N. Gol'tsman, and K. K. Berggren, Opt. Express 14, 527 (2006).

${ }^{4}$ R. E. de Lamaestre, L. Maingault, L. Frey, C. Constancias, P. Cavalier, J. C. Villegier, P. Odier, and J. P. Poizat, Proc. SPIE 7249, 72490Q (2009).

${ }^{5}$ E. F. C. Driessen and M. J. A. de Dood, Appl. Phys. Lett. 94, 171109 (2009).

${ }^{6}$ M. Tarkhov, J. Claudon, J. P. Poizat, A. Korneev, A. Divochiy, O. Minaeva, V. Seleznev, N. Kaurova, B. Voronov, A. V. Semenov, and G. Gol'tsman, Appl. Phys. Lett. 92, 241112 (2008).

${ }^{7}$ A. Divochiy, F. Marsili, D. Bitauld, A. Gaggero, R. Leoni, F. Mattioli, A. Korneev, V. Seleznev, N. Kaurova, O. Minaeva, G. Gol'tsman, K. Lagoudakis, M. Benkhaoul, F. Levy, and A. Fiore, Nat. Photonics 2, 302 (2008).

${ }^{8}$ H. Takesue, S. W. Nam, Q. Zhang, R. H. Hadfield, T. Honjo, K. Tamaki, and Y. Yamamoto, Nat. Photonics 1, 343 (2007).

${ }^{9}$ M. Halder, A. Beveratos, N. Gisin, V. Scarani, C. Simon, and H. Zbinden, Nat. Phys. 3, 692 (2007).

${ }^{10}$ E. le Coarer, S. Blaize, P. Benech, I. Stefanon, A. Morand, G. Lerondel, G. Leblond, P. Kern, J. M. Fedeli, and P. Royer, Nat. Photonics 1, 473 (2007).

${ }^{11}$ A. Verevkin, J. Zhang, R. Sobolewski, A. Lipatov, O. Okunev, G. Chulkova, A. Korneev, K. Smirnov, G. N. Gol'tsman, and A. Semenov, Appl. Phys. Lett. 80, 4687 (2002).

${ }^{12}$ A. D. Semenov, G. N. Gol'tsman, and A. A. Korneev, Physica C 351, 349 (2001).

${ }^{13}$ A. Semenov, A. Engel, H.-W. Hübers, K. Il'in, and M. Siegel, Eur. Phys. J. B 47, 495 (2005).

${ }^{14}$ P. Haas, A. Semenov, H.-W. Hübers, J. Beyer, A. Kirste, T. Schurig, K. Il'in, M. Siegel, A. Engel, and A. Smirnov, IEEE Trans. Appl. Supercond. 17, 298 (2007).

${ }^{15}$ A. Semenov, A. Engel, K. Il'in, G. Gol'tsman, M. Siegel, and H.-W. Hübers, Eur. Phys. J. Appl. Phys. 21, 171 (2003).

${ }^{16}$ J.-C. Villégier, S. Bouat, P. Cavalier, R. Setzu, R. E. de Lamaëstre, C. Jorel, P. Odier, B. Guillet, L. Mechin, M.-P. Chauvat, and P. Ruterana, IEEE Trans. Appl. Supercond. 19, 3375 (2009).

${ }^{17}$ C. Constancias, R. E. de Lamaëstre, O. Louveau, P. Cavalier, and J.-C. Villégier, J. Vac. Sci. Technol. 25, 2041 (2007).

${ }^{18}$ A. D. Semenov, P. Haas, B. Günther, H.-W. Hübers, K. Il'in, M. Siegel, A. Kirste, J. Beyer, D. Drung, T. Schurig, and A. Smirnov, Supercond. Sci. Technol. 20, 919 (2007).

${ }^{19}$ J. Kitaygorsky, J. Zhang, A. Verevkin, A. Sergeev, A. Korneev, V. Matvienko, P. Kouminov, K. Smirnov, B. Voronov, G. Gol'tsman, and R. Sobolewski, IEEE Trans. Appl. Supercond. 15, 545 (2005).

${ }^{20}$ F. S. Jelila, J.-P. Maneval, F.-R. Ladan, F. Chibane, A. Marie-de Ficquelmont, L. Méchin, J.-C. Villégier, M. Aprili, and J. Lesueur, Phys. Rev. Lett. 81, 1933 (1998).

${ }^{21}$ R. Sobolewski, A. Verevkin, G. Gol'tsman, A. Lipatov, and K. Wilsher, IEEE Trans. Appl. Supercond. 13, 1151 (2003). 